\title{
Implementation of Ethereum Blockchain in Healthcare Using IPFS
}

\author{
Neha Raut ${ }^{a}$, Dr. Kamal Shah ${ }^{b}$ \\ Thakur College of Engineering and Technology \\ Kandivali, India ${ }^{\mathrm{a}, \mathrm{b}}$ \\ neharaut281997@gmail.com ${ }^{\mathrm{a}}$, kamal.shah@thakureducation.org b
}

\begin{abstract}
Blockchain technology is rapidly gaining attention towards the Security of confidential data. The healthcare industry is one of the fields of organization where high risk involves $\&$ its attracted attention of many technological organizations so this field required the security for securing their data. By using the Blockchain technology there are numerous opportunities for healthcare industry to achieve \& gain. Such as reduced transaction costs, increased transparency for regulatory reporting, efficient healthcare data management and healthcare records universality as well as able to access data from any location. In the context of smart healthcare system Blockchain may provide distinct benefits, particularly from a context-aware perspective where efficient and personalized solutions may be provided to citizens and the society our main concern in this proposed methodology is to protect patient's data effectively as well patients must have rights to preserve their records. Patients have confidentiality towards his/her record. Ethereum blockchain implementation is done in this system. Providing Eth to store patient's record in blockchain is quite expensive. For this purpose IPFS (InterPlanetary File System) implemented along with Ethereum blockchain.
\end{abstract}

Keywords: blockchain, ethereum, healthcare, IPFS, smart contract, secure healthcare system.

\section{Introduction}

Blockchain is a technology that can solve a variety of problems, such as ten birds with one bullet. The financial sector, supply chain management, food industry, energy sector, internet of things, and healthcare are all using blockchain as a safe and reliable platform for secure data sharing 1 . Blockchain is a decentralised framework that solves the issues that centralised systems have. When it comes to applications, blockchain integrates peer-to-peer networks with cryptographic algorithms to make it safe and stable 2. A blockchain is a set of sequentially ordered records organised in a block structure. A hash (digital fingerprint or unique identifier) is included in each data block, as well as timestamped batches of recent transactions and a hash of the previous block 3, 4. Blockchain is a method of storing data in such a way that it is difficult or impossible to alter, hack, or trick it. A blockchain is a decentralised ledger of transactions that is duplicated and distributed through the blockchain's entire network of computer systems. Each block in the chain contains a number of transactions, and if a new transaction occurs on the blockchain, a record of that transaction is added to the ledger of each party. Distributed Ledger Technology is a decentralised database that is run by multiple participants (DLT). This means that if a single block in a chain is modified, it will be instantly clear that the chain has been tampered with 5. Hackers would have to alter every block in the chain, through all distributed versions of the chain, if they wanted to corrupt a blockchain system. Each block 
International Journal of Intelligent Communication, Computing and Networks Open Access Journal (ISSN: 2582-7707)

https://doi.org/10.51735/ijiccn/001/17

is linked in sequential order in this design, and the connected blocks are referred to as a blockchain. Modifying one of the blocks in the middle of the chain is virtually impossible since all of the blocks following the changed block must be modified at the same time. The data on the blockchain network is permanent 6 . A smart contract is a computer protocol that runs automatically when the prerequisites are met, and it is an entity separate from the original blockchain technology. In this proposed Healthcare Management System main aim is to add patient's records on the bloackchain securely so that medical history can be maintained as well as patient will definetly feel safe about their medical history 8 . Nobody can make any changes to his / her records. Ethereum blockchain implementation is done along with IPFS.

\subsection{Literature Review}

M. A. Lambey et al., (2020) conducted a thorough investigation into Big Healthcare Data. Discuss how you can improve patient outcomes, predict infectious outbreaks, gain valuable information, avoid preventable infections, lower health-care costs, and improve overall quality of life. However, deciding on the best use of data while maintaining patient confidentiality and the right to privacy is a challenging job. The limitations of current technology must be identified, and guidelines for future research must be considered 1 .

M. Zalloum et al., (2020) uses the traditional health system's concept of e-Healthcare. However, current e-Healthcare systems are not yet fully developed and mature, and therefore lack the level of confidentiality, integrity, anonymity, and user trust necessary for widespread adoption. Patient interest in the healthcare sector and the quality of healthcare services are two important aspects of any functioning healthcare business. Addressing privacy concerns necessitates addressing security issues such as access control, authentication, non-repudiation, and transparency, without which end-to-end privacy is difficult to achieve 2 .

Koosha Mohammad Hossein et al., (2019) propose a blockchain-based architecture for e-health applications that offers an effective access control system while maintaining privacy. By taking advantage of Blockchain (BC) special features, such as immutability and user confidentiality, while changing the traditional blockchain structure to address IoT application problems (low throughput, high overhead, and latency) 3.

Farhad Ahamed et al., (2018) propose personalized healthcare ( $\mathrm{PH})$ is a radical patient-centered approach to healthcare that aims to change the current system. Patient data from electronic health records (EHR), Internet of Things (IoT) sensor devices, wearables and mobile devices, web-based knowledge, and social networking is the subject of this new advancement. To develop disease progression approaches, disease detection, patient self-management, and clinical intervention, $\mathrm{PH}$ applies Artificial Intelligence (AI) techniques to the collected dataset. In order to create analytic models, machine learning techniques are often used 4.

Dash et al., (2019) propose a healthcare-focused architecture, in which hospital records, patient 
International Journal of Intelligent Communication, Computing and Networks Open Access Journal (ISSN: 2582-7707)

https://doi.org/10.51735/ijiccn/001/17

medical records, medical exam reports, and Internet of Things devices are all sources of big data. Biomedical science generates a large amount of Big Data relevant to public health. This data must be carefully managed and analysed in order to obtain valuable information. Otherwise, identifying a needle in a haystack by examining big data is akin to finding a needle in a haystack. Discuss also by offering early signs of sickness symptoms and helping to identify new biomarkers and smart methods for clinical action to enhance the quality of life 5.

K.M. Hossein et al., (2019) described Blockchain as a form of database used to store data in a distributed system. The differences between Blockchain and Bitcoin were also discussed, with a focus on future work on using Blockchain technology for electronic health records. It will look into how a hospital or health authority can use or request a patient's medical records from a third party (external stakeholder) without jeopardising the patient's privacy 6 .

S. Pariselvam et al., (2019) proposed that difficulties and different protection methods for protecting the privacy of health records in the cloud are discussed. A new scheme based on DES was proposed for preserving health records based on challenges and different securities. This scheme allows for powerful and privacy-preserving indexes to be encrypted using different symmetric keys, and encrypted data from multiple providers to be merged in the cloud without the content being known. It also offers stable database processing, allowing users to send a single data query to the cloud without knowing the contents 7 .

V. Ramani et al., (2019) says that one of the most important considerations of today's smart healthcare systems is the security of confidential patient data from possible attackers. As a result, it's critical to have safe data access systems in place to ensure that only approved parties have access to a patient's medical records. The proposed framework is also capable of protecting patients' privacy. Our scheme's security review reveals that it can withstand well-known attacks while retaining system integrity. But the problem here is data will be on centralized server 8 .

N. Rifi et al., (2017) says that patients and approved users of this vital medical data are concerned about the privacy and confidentiality of medical information. Scalability and interoperability, on the other hand, are critical issues that must be addressed in the final solution. Author illustrates the basic issues and benefits of blockchain technology for deploying a reliable and scalable solution for medical data sharing in order to achieve the best possible output 9 .

X. Liang et al., (2017) A mobile application is used to capture health data from wearable devices, manual reporting, and medical devices, then coordinate the information to the cloud for sharing with healthcare providers and insurance companies. To maintain the integrity of health records, a proof of integrity and authentication is permanently retrievable from the cloud database and anchored to the blockchain network for each record 10. 


\subsection{Problem Statement}

Due to its decentralization, immutability, accountability, and traceability functionality, blockchain is a viable technology that can enhance the healthcare data sharing and storing method. Data tampering is one of the most serious problems in current technology. Although it may be possible to detect and forecast patients' states using data analytics within a single entity, handling and correlating patients' related data across various organizations is difficult. The issue isn't a lack of resources rather, it's a lack of resource management. The patient may have some way in how their medical data is used and shared by physicians. Any party interested in obtaining a patient's medical data may use the Blockchain to obtain the necessary permission. Medical records created and stored on the blockchain would be completely secure

\subsection{Use of Blockchain in Healthcare}

Several blockchain applications in the healthcare sector have shown that the platform can be beneficial in a number of ways. The encryption algorithms known as hash algorithms check each sequence of transactions known as a block inside the blockchain, which is perhaps the most significant advantage of blockchain. Before starting a new transaction, each previous transaction block is reviewed. Since hash algorithms can't be changed until the transaction is encrypted, each transaction within the blockchain is guaranteed to be valid. The blockchain's transactions are also permanent, ensuring that such transactions cannot be reversed or modified. If a record needs to be updated, a new record must be created, which is why the blockchain is referred to as an append-only ledger. Furthermore, all blockchain transactions are time-stamped, which improves transaction transparency and accountability 7. In this scenario, healthcare stakeholders will monitor how and when healthcare information is used. Furthermore, in a situation where one node is compromised within the blockchain, the entire ledger is not affected because the information within the ledger is spread through several nodes within the distributed network. Blockchain is permanent and, thus, the health records of patients can not be tampered with during transmission. Because blockchain uses public private keys to encrypt data, when personal health records are exchanged between healthcare stakeholders, patient identities can not be disclosed. Moreover, blockchain allows a patient to determine how to use or share his/her data through smart contracts 10 .

\subsection{Motivation}

1) The patient can have some control over how their medical data gets used and shared by the Doctors.

2) Any party which is looking to get the medical data about a patient could check with the Blockchain to get the necessary permission.

3) Medical record that has been generated and added to the blockchain will be completely secure 


\subsection{Objective}

1) Health Data Accuracy :- Blockchain medical records are possible when Blockchain and healthcare are combined. The precision with which treatments are administered can be greatly enhanced.

2) Health Data Interoperability :- Any health-care facility could benefit from digital medical records backed by Blockchain technology regardless of where the facility is located.

3) Health Data Security :- A Blockchain's data records are unchangeable (unchangeable after they are input). This reduces the chances of data manipulation or mismanagement. Since there is no single point of failure, health data stored on a Blockchain is safe from natural disasters.

4) Health Data Handling Costs :- Data is well-kept in a Blockchain architecture and can be easily accessed by relevant Doctors at any time with patient's permission.

\subsection{Issues and Challenges}

Data tampering is one of the most serious problems in current technology. Although it may be possible to detect and forecast patients' states using data analytics within a single entity, handling and correlating patients' related data across various organisations is difficult. The issue isn't a lack of resources rather, it's a lack of resource management.

\subsection{Security and Privacy analysis}

We look at how well the proposed architecture performs in terms of security and privacy. We investigate the CIA security triad of our architecture to demonstrate its durability against multiple attacks (Confidentiality, Honesty, and Availability). First and foremost, we are addressing aspects of the CIA triad in the context.

- Confidentiality :- Only authorised users have access to the messages, according to confidentiality. We encrypt communication between modules to ensure confidentiality, ensuring that user data (generated by sensors) is protected from unauthorised users sniffing it.

- Integrity :- Flow management for patients Patients' past medical test results enable the healthcare system to anticipate patient flow.

- Availability :- BC ensures the data is available at all times.

\subsection{Proposed System}

Main aim of proposed system is to protect patient's data from different illegal access. Maintaing data after many years is not possible so access of histical reports is also possible.

1) It is not possible for patient to carry all of his data all the time. To maintain data patient must have to register on blockchain address.

2) Patient goes to hospital ' $X Y Z$ ' for treatment. If doctor wants to upload patient's document then approvalfrom patient is must. That means full data control is in patients hand controlling data 
privacy.

3) Every time it is necessary to send approval request to the patient.

4) Here, In hospital ' $A B C$ ', patient needs to share his first report ,he/she will just have to provide own address and the hospital 'ABC' can access that particular record.

5) The patients on login into his account and can see the Transactions for every report.

6) Patient cannot upload own reports because it would lead to uploading fake reports.

\subsection{Proposed Methdology Architecture}

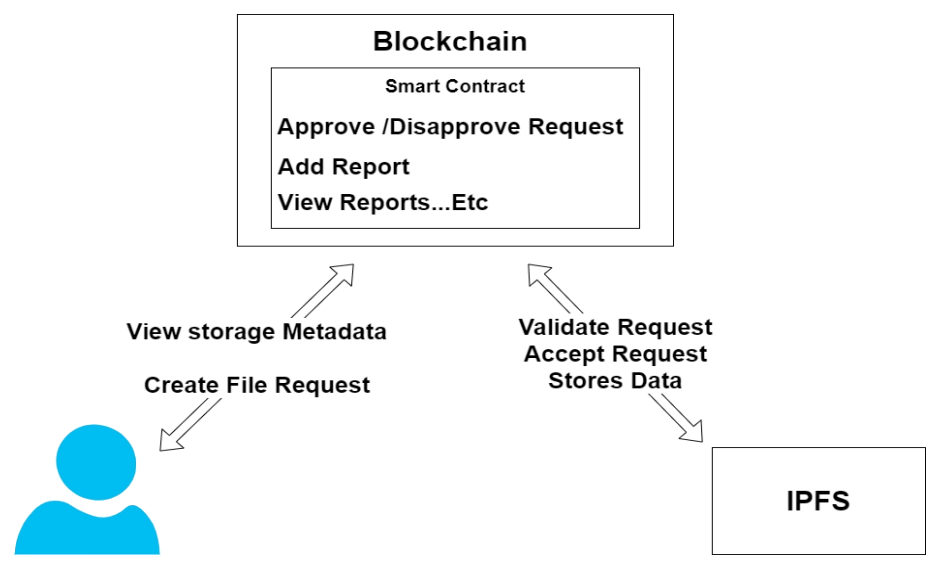

Figure 01: Block Diagram of Proposed System

- Ethereum Blockchain :-

Ethereum is an open-source, blockchain-based, decentralised software platform that was started in 2015 and is used by its own cryptocurrency, ether. It enables SmartContracts and Distributed Applications (Apps) to be developed and run without any downtime, fraud, control, or third-party intervention.

- Smart Contracts : -

The term "smart contract" refers to a programme that runs on the Ethereum blockchain. It's a list of code (its functions) and data (its state) stored on the Ethereum blockchain at a specific address.

- IPFS :-

The InterPlanetary File System (IPFS) is a distributed file system protocol and peer-to-peer network for storing and sharing data. In a global namespace linking all computing devices, IPFS uses content-addressing to uniquely define each file.

\section{Implementation}

System Implemenation is done using Ethereum Blockchain. With the help of Ganache Ethereum is implemented. Ganache is a tool for creating a private Ethereum blockchain to test your Solidity contracts on.

- Ethereum Blockchain :- The healthcare Blockchain smart contract framework has been implemented using Ethereum. This is an open-source network that is currently one of the largest 
public blockchain networks, with a wide public DApp repository and an active community.

- Dapps :- A decentralised application (dapp) is an application that incorporates a smart contract and a frontend user interface and is designed on a decentralised network. Smart contracts in Ethereum are as available and transparent as open APIs dapp can also have a smart contract written by someone else.

- Ganache :- Ganache is a blockchain that is tailored to the needs of Ethereum developers. It can be used to monitor the chain's operation while running tests, executing commands, and inspecting states.

- Smart Contract :- Solidity is an object-oriented programming language designed specifically for contract writing. It's a high-level programming language that borrows features from $\mathrm{C}++$, Python, and JavaScript. The Solidity compiler converts your source code to bytecode, which is then executed on the Ethereum Virtual Machine (EVM).

- Metamask :- Metamask is a browser-based cryptocurrency wallet that works with Chrome, Firefox, and Brave. It's also available as a browser add-on. This means it acts as a connection between standard web browsers and the Ethereum blockchain.

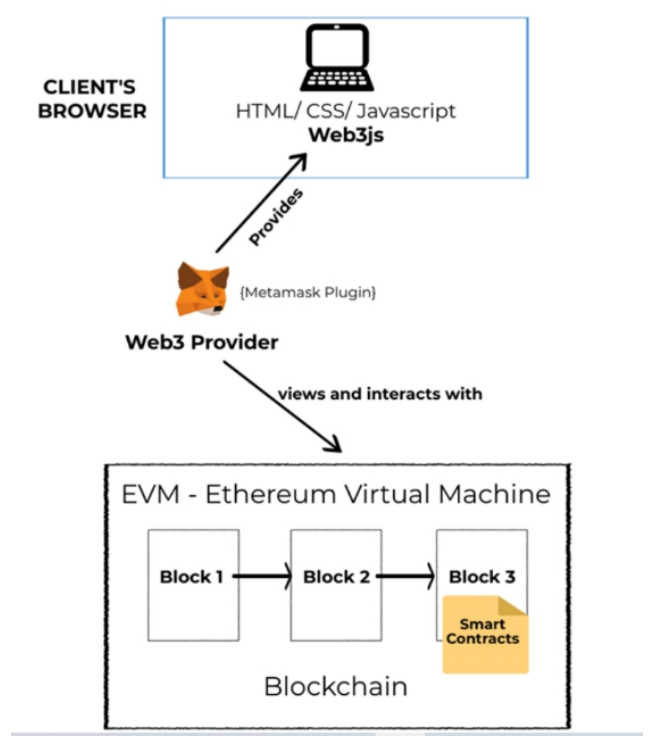

Figure 02: Working Model

Implemetation is done on ethereum Blockchain. With the help of Ganache Truffle, Metamask system is build. In our system we are going to store patient's record that might be any any format like pdf, jpg, png....etc. As we have implemented ethereum blockchain in Ganache it is not possible to store huge document format as it is directly proportional to eth cost. To solve this problem IPFS is used. With the help of IPFS direct document will store on IPFS and its hash value on blockchain, which will eventually reduce cost of the system.

1) Client's Browser :- 
International Journal of Intelligent Communication, Computing and Networks Open Access Journal (ISSN: 2582-7707)

https://doi.org/10.51735/ijiccn/001/17

It functions similarly to a standard browser for any html, CSS, or javascript-based web application.

2) Web3.js :-

Web3.js is a collection of libraries that allows your browser to communicate with blockchain. It allows you to read and write data from smart contracts, as well as pass ethers between accounts.

3) Web3 Provider :-

The Ethereum network is made up of nodes, each of which has the same copy of data. In web3.js, specifying a "web3 provider" tells our code which node we'll read and write data from. In our implementation, we use Metamask, which injects its web3 provider into the browser. Metamask is a Chrome and Firefox browser extension that allows users to securely manage their Ethereum accounts and private keys, as well as use these accounts to communicate with Web3.js-enabled websites.

4) Ethereum Virtual Machine :-

Every ethereum node in the Ethereum network has its own EVM implementation, which is responsible for running the same smart contract instructions across the Ethereum network.

We have to deploy smart contracts with etherum blockchain for which Ganache and metamask is there. By connecting all of these modules we are making a system which is very useful for storing patient's historical medical history along with some protocols which are written as smart contract.

Home screen will be appearing like this
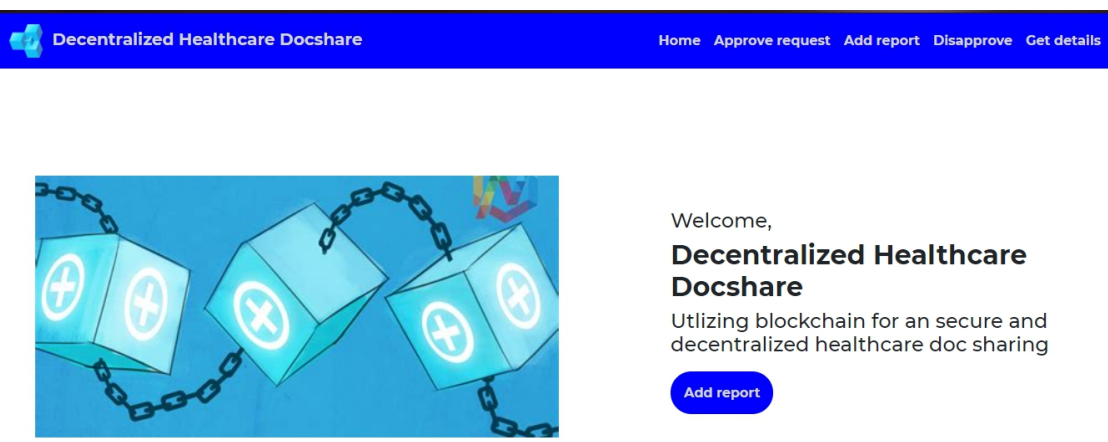

Welcome,

Decentralized Healthcare

Docshare

Utlizing blockchain for an secure and

decentralized healthcare doc sharing

Add report

Figure 03: Home page

- Patient must have to approve Doctor Otherwise Doctor cannot access patient's previous medical records. For that purpose patient should approve Doctor's address in order to give access. 
Figure 04: Approve Doctor

- Once Patient approves the Doctor then patient's new records can be added by the Doctor. New medical report can be anything like MRI, blood report, X-rays, prescriptions....etc. It can be document, pdf, image anything. For this purpose Doctor have to add patients address and necessary information

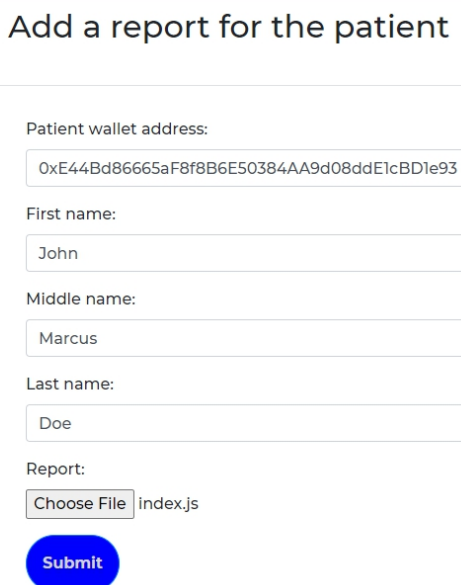

Figure 05: Add Report

- Sometimes Doctor just wants to see patient's previous medical history to give him proper treatment. This can be possible if patient approves Doctor. Every time Doctor have to ask for approval otherwise Doctor will access patients record for long time and confidentiality and privacy of patients data will not be maintained. 
Patient details:

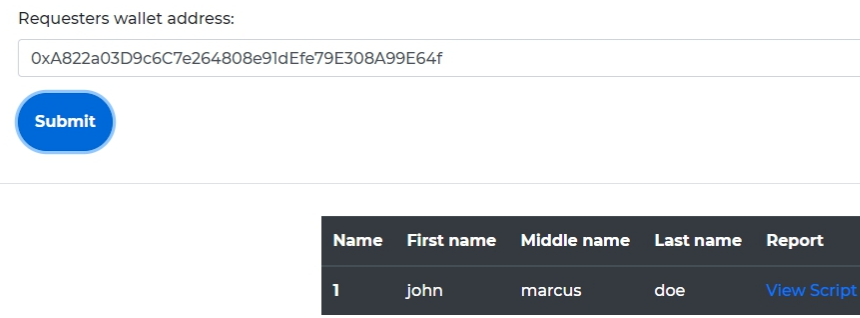

Figure 06: Patients Details

- If patient will think that he/she does not want Doctor to access his medical history anymore then Patient can disapprove that Doctor with the help of his address

The Ethereum blockchain can be used to store images. However, it is not recommended that to do so. This is due to the high cost of storing images on the Ethereum blockchain. If we can only store a few kilobytes of data on the blockchain, we'll still need to rely on a centralised server. Fortunately, the InterPlanetary File System IPFS) provides a solution for storing data on a decentralised network. Using DApp to upload a document to IPFS and then store the IPFS hash on the Ethereum blockchain. The user will receive a transaction receipt after the IPFS hash number is sent to the Ethereum blockchain. We'll build a front-end using the Create-React-App platform. Anyone who has MetaMask installed in their browser can use this Dapp. With the use of IPFS cost of system is reduced.

\section{Experimental Results}

Table 1: Feature analysis on various parameters.

\begin{tabular}{|l|c|c|c|c|}
\hline Features & 4 & 6 & 8 & Proposed System \\
\hline Availability & Yes & Yes & Yes & Yes \\
\hline Decentralized & No & Yes & Yes & Yes \\
\hline
\end{tabular}


International Journal of Intelligent Communication, Computing and Networks Open Access Journal (ISSN: 2582-7707)

https://doi.org/10.51735/ijiccn/001/17

\begin{tabular}{|l|c|c|c|c|}
\hline Data Privacy & Yes & Yes & Yes & Yes \\
\hline Patient Centric & Partially & No & No & Yes \\
\hline Immutability & Partially & Yes & Yes & Yes \\
\hline Integrity & Yes & Yes & Yes & Yes \\
\hline Trustful & Partially & Yes & Yes & Yes \\
\hline Storage Flexibility & Yes & No & Partially & Yes \\
\hline
\end{tabular}

1) Availability :-

System availability is must as healthcare service is $24 / 7$.

2) Decentralized :-

System storage and management activities are controlled by several local authorities than single one. Blockchain is decentralized storage as data is stored on blocks.

3) Data Privacy :-

Our access management scheme ensures data privacy and ownership of individuals by using the security capabilities of blockchain and smart contracts. Malicious access is prevented by the user's ability to identify him and the smart contract's authorization.

4) Patient centric :-

All the authorities regarding sharing and uploading data must be handled by patients. In our proposed system patient will decide about all the necessary approvals and disapprovals of Doctors.

5) Immutability :-

Once data will be uploaded it should not be changed. Once the data will be uploaded on blockchain it cannot be changed.

6) Integrity :-

Integrity ensures that patient data is exchanged between approved users in an unaltered state. As blockchain is immutable maintaining integrity is possible.

7) Trustful :-

Every authority is on the patient's hand that will make our system trust worthy.

8) Storage Flexibility :-

We are storing data on the IPFS which reduces costs of ethereum blockchain.

Patient's medical records are stored on the Blockchain. But storing such a huge data directly on the blockchain is very costly as a result we have implemented IPFS to solve this problem.

Patient will decide who can access his data. Once patient gives the permission to particular Doctor that means patients gives all the required permission to Doctor for uploading and accessing patient's previous history.

\section{Discussion}

Blockchain gives upmost security and privacy to the patient's data. Patients can give approval to the Doctor to see his records, access previous documents as long as patient wants doctor to do so. Now 
International Journal of Intelligent Communication, Computing and Networks Open Access Journal (ISSN: 2582-7707)

https://doi.org/10.51735/ijiccn/001/17

patients should not have to be worried about carrying whole set of document or no one including patient alters the data or deletes it once uploaded. On blockchains, large files cannot be stored efficiently. On the one hand, the blockchain becomes bloated with data that must be propagated around the network. However, since the blockchain is replicated on several nodes, a large amount of storage space is needed without serving an immediate purpose, especially if the node operator does not need to access every file stored on the blockchain. Furthermore, since more data needs to be processed, exchanged, and stored, the cost of running blockchain nodes rises. IPFS is a file sharing system that can be used to store and transfer large files more effectively. It is based on cryptographic hashes, which can be stored easily on a blockchain. Etherum cost is more which can be reduced by this technique. As well as smart contracts are running to give most security.

Proposed system is developed with the help of ethereum blockchain which stores patient 's related data on IPFS. Now, privacy of patient's data is increases. Whole control is on the patient's hand. Model is patient centric. Patient can approve or disapprove the doctor as well as allow him/ her to see previous histories and add new records. Use of IPFS increases its capability to store large amount of data. In future system can also arrange appointments, bookings, payments and insurance.

\section{Acknowledgments}

I would like to take opportunity to express my sincere thanks to my guide Dr. Kamal Shah, Professor \& Dean, TCET for her keen interest, inspiring guidance, constant encouragement with my work during all stages, to bring this research into fruition.

\section{References}

1. M. A. Lambay and S. Pakkir Mohideen, "Big Data Analytics for Healthcare Recommendation Systems," 2020 International Conference on System, Computation, Automation and Networking (ICSCAN), 2020, pp. 1-6, doi: 10.1109/ICSCAN49426.2020.9262304.

2. M. Zalloum and H. Alamleh, "Privacy Preserving Architecture for Healthcare Information Systems," 2020 IEEE International Conference on Communication, Networks and Satellite (Comnetsat), 2020, pp. 429-432, doi: 10.1109/Comnetsat50391.2020.9328985.

3. K. M. Hossein, M. E. Esmaeili, T. Dargahi and A. khonsari, "Blockchain-Based Privacy-Preserving Healthcare Architecture," 2019 IEEE Canadian Conference of Electrical and Computer Engineering (CCECE), 2019, pp. 1-4, doi: 10.1109/CCECE.2019.8861857.

4. F. Ahamed and F. Farid, "Applying Internet of Things and Machine-Learning for Personalized Healthcare: Issues and Challenges," 2018 International Conference on Machine Learning and Data Engineering (iCMLDE), 2018, pp. 19-21, doi: 10.1109/iCMLDE.2018.00014.

5. Dash, S., Shakyawar, S.K., Sharma, M. Big data in healthcare: management, analysis and future prospects. J Big Data 6, 54 (2019). https://doi.org/10.1186/s40537-019-0217-0

6. K. M. Hossein, M. E. Esmaeili, T. Dargahi and A. khonsari, "Blockchain-Based Privacy-Preserving Healthcare Architecture," 2019 IEEE Canadian Conference of Electrical and Computer Engineering (CCECE), 2019, pp. 1-4, doi: 10.1109/CCECE.2019.8861857.

7. S. Pariselvam and M. Swarnamukhi, "Encrypted Cloud Based Personal Health Record Management Using DES Scheme," 2019 IEEE International Conference on System, Computation, Automation and Networking (ICSCAN), 2019, pp. 1-6, doi: 10.1109/ICSCAN.2019.8878773. 
International Journal of Intelligent Communication, Computing and Networks

Open Access Journal (ISSN: 2582-7707)

https://doi.org/10.51735/ijiccn/001/17

8. V. Ramani, T. Kumar, A. Bracken, M. Liyanage and M. Ylianttila, "Secure and Efficient Data Accessibility in Blockchain Based Healthcare Systems," 2020 IEEE Global Communications Conference (GLOBECOM), 2018, pp. 206-212, doi: 10.1109/GLOCOM.2018.8647221.

9. N. Rifi, E. Rachkidi, N. Agoulmine and N. C. Taher, "Towards using blockchain technology for eHealth data access management," 2017 Fourth International Conference on Advances in Biomedical Engineering (ICABME), 2017, pp. 1-4, doi: 10.1109/ICABME.2017.8167555.

10. X. Liang, J. Zhao, S. Shetty, J. Liu and D. Li, "Integrating blockchain for data sharing and collaboration in mobile healthcare applications," 2019 IEEE 28th Annual International Symposium on Personal, Indoor, and Mobile Radio Communications (PIMRC), 2017, pp. 1-5, doi: 10.1109/PIMRC.2017.8292361. 